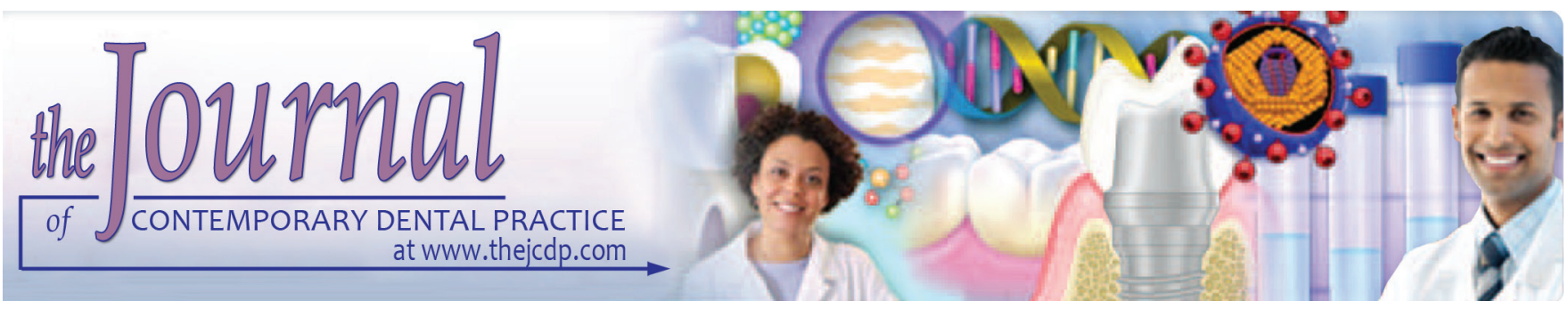

\title{
Oral Health Status in Alzheimer's Disease Patients: A Descriptive Study in an Italian Population
}

\author{
${ }^{1}$ Giovanni D'Alessandro, ${ }^{2}$ Tommaso Costi, ${ }^{3}$ Nadia Alkhamis, ${ }^{4}$ Simone Bagattoni, ${ }^{5}$ Agnese Sadotti, ${ }^{6}$ Gabriela Piana
}

\begin{abstract}
Aim: To evaluate the oral health status in Alzheimer's disease (AD) patients.

Materials and methods: A descriptive study was performed on 120 AD patients (60 institutionalized in a public institute and 60 attended a daytime center), from September 2013 to January 2014. About 103 subjects formed the control group. The following medical and dental data were collected: dementia severity, pharmacological therapy, physical status (American Society of Anesthesiologists $[A S A])$, decayed $(D)$, filled $(F)$, and remaining natural teeth $(T), D F / T$ ratio, community periodontal index $(\mathrm{CPI})$, and gingival index (GI). A t-test for independent samples and the Spearman's correlation test were used to evaluate all variables. The significance level was set at 0.05 .
\end{abstract}

Results: Statistically more AD patients (91.7\%) were under pharmacological therapy and their physical status was more severe (ASA 2, ASA 3) compared with control subjects $(p<0.001)$. Moreover, they presented numbers of D, CPI, and $G$ I significantly higher $(p \leq 0.005)$. In the institutionalized subgroup, statistically more moderate and severe $A D$ cases were detected and more patients were edentulous $(p<0.001)$. Noninstitutionalized patients presented DF/T ratio, CPI, and GI significantly lower $(p \leq 0.024)$. A significant weak negative correlation ( $r=-0.121$ to -0.372 ) between epidemiologic indices and $A D$ severity was observed.

Conclusion: Alzheimer's disease patients show a low oral health status that decreases progressively as the disease severity aggravates. Therefore, further studies are necessary to investigate oral health care interventions for $A D$ patients.

\footnotetext{
${ }^{1,2,4-6}$ Department of Biomedical and Neuromotor Sciences Dental School, Alma Mater Studiorum, Università di Bologna Bologna, Italy

${ }^{3}$ Department of Biomedical and Neuromotor Sciences, Dental School, Alma Mater Studiorum, Università di Bologna, Bologna, Italy; Department of Pediatric Dentistry and Orthodontics, King Saud University, Riyadh, Kingdom of Saudi Arabia

Corresponding Author: Giovanni D'Alessandro, Department of Biomedical and Neuromotor Sciences, Dental School, Alma Mater Studiorum, Università di Bologna, Bologna, Italy, Phone: +390512088150, e-mail: dr.dalessandro@gmail.com
}

Clinical significance: It would be beneficial to introduce trained professional figures in specialized elderly institutions for regular follow-up visits and professional oral hygiene procedures. This task has to be coordinated with the treating physician, family members, and/or caregivers. Knowing that the severity of $A D$ has a negative effect on the oral health status and the type of institutionalization exacerbates it.

Keywords: Alzheimer's disease, Dementia, Descriptive study, Oral health, Professional.

How to cite this article: D'Alessandro G, Costi T, Alkhamis N Bagattoni S, Sadotti A, Piana G. Oral Health Status in Alzheimer's Disease Patients: A Descriptive Study in an Italian Population. J Contemp Dent Pract 2018;19(5):483-489.

Source of support: Nil

Conflict of interest: None

\section{INTRODUCTION}

As the world population is becoming progressively older, the interest of health care professionals toward the quality of elderly life is increasing. One of the most studied consequences of aging is dementia.

Dementia is caused by different disorders that affect cerebral structures and functions, causing progressive deterioration of memory, other mental functions, and behavior. ${ }^{1}$ The prevalence of dementia for people aged $>60$ years varied in a narrow band, 5 to $7 \%$ in most world regions. It was estimated that 35.6 million people lived with dementia worldwide in 2010 , with numbers expected to almost double every 20 years, to 65.7 million in 2030 and 115.4 million in 2050. ${ }^{2}$ Alzheimer's disease is generally considered the most common dementia subtype, accounting for between 60 and $80 \%$ of dementia cases. $^{3-5}$

The incidence rate for AD increases exponentially with age, with the most pronounced increase occurring through the 7 th and 8 th decades of life. There is evidence that in Western society prevalence and increase display a 
cohort effect with later-born individuals having a lower risk than those born earlier in the past century. ${ }^{6-9}$

The etiology of AD is multifactorial and involves genetic and environmental risk factors, such as family history, APOE- $\varepsilon 4$ allele, Down's syndrome, advanced age, lower educational level, traumatic brain injury, cerebrovascular disease, blood pressure, type II diabetes, body weight, plasma lipid levels, metabolic syndrome, and smoking. ${ }^{10}$

However, up to now, the etiology of AD is still not fully clear; however, inflammation of the cerebral tissues for sure plays an important role in the insurgence of this pathology. For these reasons, chronic inflammation in periodontal disease has been suggested as a potential risk factor in AD. Initial data demonstrate elevated antibodies to periodontal disease bacteria in subjects years prior cognitive impairment and suggest that periodontal disease could potentially contribute to the risk of AD onset/ progression. ${ }^{11}$ However, a 2016 review highlights that even if the data suggest a positive association between periodontitis and dementia, the power of this association and the presence of relation between them is unknown due to the heterogeneity of the definition of both diseases, the methods of the study analyzed, and the variety of the physiopathological mechanism involved. ${ }^{12}$ An association between AD and various infectious agents (herpes simplex 1, Chlamydophila pneumoniae, and Spirochetes) was also hypothesized. ${ }^{13}$

The pharmacological treatment of AD is usually symptomatic. Treatment guidelines for AD state, as the first line of therapy, acetylcholinesterase inhibitors, which are effective in delaying cognitive impairment. In addition, adjunctive medications are used, such as antioxidants, antidepressives, anxiolytics, hypnotics, and antipsychotics. Frequently, these medications have adverse effects that give oral manifestations; the most effective on oral health is xerostomia. ${ }^{14}$ Other important oral manifestations include soft tissue lesions, allergic reaction, altered taste sensation, gingival hyperplasia, burning mouth, dental caries, and involuntary oral movements, in addition to osseous remodeling of the alveolar bone.

Dental and periodontal infections are common in AD patients and dentists should focus on this topic for several reasons: domiciliary oral hygiene is strongly compromised because learning and attention processes are seriously injured. Patients could also forget to brush their teeth because of the loss of memory, a classic sign of AD. ${ }^{15}$

In fact, $\mathrm{AD}$ patients present a high prevalence of decayed, missing, and filled teeth (DMFT) and periodontal disease affected around $87.2 \%$ of the remaining teeth. In addition, the patients with more missing teeth are older than the ones with a more complete, but unhealthy, dentition, correlating the incidence of oral diseases with the progression of $\mathrm{AD} .{ }^{15}$ As the severity of $\mathrm{AD}$ increases, the salivary flow of major salivary glands is reduced.

The aims of this study were to compare the oral health status in two samples composed respectively, of institutionalized and noninstitutionalized AD patients and to compare them with a control group of elderly subjects without dementia. In addition, the correlation between the stage of dementia and the oral health status of this population was investigated.

\section{MATERIALS AND METHODS}

\section{Study Population}

One hundred and twenty (83 females, 37 males) AD patients participated in the study. The main study group was divided into two subgroups: 60 were institutionalized in a public elderly institute "Istituto Giovanni XXIII" in Bologna, Italy; while 60 were not institutionalized and only attended a daytime recreational center "Amarcord Cafe/A.S.P, casa Valloni" in Rimini, Italy. The data collection took place from September 2015 to January 2016. The selection criteria for the study sample were a confirmed diagnosis of $\mathrm{AD}$ and age older than 65 years.

A control group of 103 subjects (60 females and 43 males) was used for comparison with the following inclusion criteria: negative medical history for dementia and age greater than 65 years.

This study is an observational, cross-sectional comparative study. All participants were native Italian speakers and belonged to an Italian ancestry. Signed informed consents were obtained from the participants or their legal guardians. The study was approved by the Sant'OrsolaMalpighi Hospital Research Ethics Committee (Bologna, Italy) (N. 0019293 in 20/09/2013).

\section{Data Collection}

The medical and dental history data were collected from the medical records of the patients and/or interviewing the patients and their family members/caregivers. The following data were registered for each patient: gender, age, age at diagnosis of $A D$, severity of dementia according to the Clinical Dementia Rating (CDR) score, presence or absence of systemic pathologies, the physical status according to ASA.

Clinical examination was performed for each patient by a single experienced operator, with the patient sitting on a chair (or, when necessary, on a wheelchair) under a good light source using a dental mirror, a dental explorer, and a World Health Organization (WHO)-type periodontal probe. 
The following clinical data were registered: dental chart, DMFT, present caries experience (DF/T index), which relates the number of carious and filled teeth to the present natural teeth; in both DMFT and DF/T, crowned teeth were considered filled teeth; the CPI and the GI of Silness-Löe were recorded as recommended by the codes and criteria of the WHO. ${ }^{16}$

No radiographs were taken. The first author performed the data collection in order to simplify the operational process. Intraexaminer reliability was measured by examination of a random sample of 30 patients 2 weeks after the initial examination. Weighted (Fleiss-Cohen) Kappa statistics were used to confirm the examiner calibration and to assess intraexaminer reliability: the percentage of agreement and the Kappa statistic were $97 \%$ and 0.95 respectively.

\section{Statistical Analysis}

The data were entered in MS Excel and analyzed using the Statistical Package for the Social Sciences version 14.0 statistical software (IBM Corporation, NY, USA). Descriptive data were calculated with median values, range, frequencies, and percentages for nonparametric data (qualitative, categorical, and nominal variables), while means and standard deviations were used as the quantitative variables with a normal distribution.

A t-test for independent samples was used to determine differences between the control group and the total AD group. The same test was used to compare the institutionalized $\mathrm{AD}$ and noninstitutionalized $\mathrm{AD}$ subgroups. The Spearman's correlation test (r) was performed for inference of correlations between ordinal variables. Statistical significance levels for all tests were set at $\mathrm{p}<0.05$.

\section{RESULTS}

Table 1 shows the characterization of both the control and $\mathrm{AD}$ groups and a t-test showing the differences between the two groups. The age is displayed as the median values and range: in the control group the age was 77.68 years (min: 65, max: 94) in which $58.3 \%$ are females and $41.70 \%$ are males. The median age of AD group was 79.1 (min: 65 , max: 101 ) in which $69.2 \%$ was females and $30.8 \%$ was males. Male and female subjects were equally distributed in both $\mathrm{AD}$ and control groups $(\mathrm{p}=0.436, \mathrm{p}=0.990)$. The age of subjects did not differ between the two groups $(p=0.940)$.

Medical data are also shown in Table 1. In the control group, 43 patients $(41.70 \%)$ did not report systemic diseases, while $60(58.30 \%)$ reported at least one systemic disease. These percentages were not statistically different for the AD group in which 71 patients (59.20\%) manifested additional systemic pathologies, while $49(40.80 \%)$ was negative for systemic illnesses $(p=0.860$ and $p=0.841$ respectively). A significantly larger percentage of $\mathrm{AD}$ patients $(91.7 \%)$ were under pharmacological therapy compared with control subjects $(59.2 \%, \mathrm{p}<0.001)$. In the AD group, 58 patients were classified as ASA $1(56.30 \%)$, 40 patients as ASA $2(38.80 \%)$, and 5 as ASA 3 (4.90\%). The physical status of AD patients was more severe in all three categories (ASA 1, ASA 2, and ASA 3) $(\mathrm{p}<0.001)$. The percentage of edentulism in AD patients (39.16\%) was not significantly different than in the control group $(31.6 \%)(\mathrm{p}=0.102)$.

Epidemiological indices for both control and $\mathrm{AD}$ groups and a t-test showing the differences between the two groups are reported in Table 2. The values were calculated only for dentate subjects (71 control subjects and $73 \mathrm{AD}$ subjects). Statistically significant differences were

Table 1: Characteristics of the control and AD group

\begin{tabular}{|c|c|c|c|c|}
\hline & & Control $(n=103)$ & $A D(n=120)$ & Significance \\
\hline \multirow[t]{2}{*}{ Gender } & Female & $58.30^{*}$ & $69.20^{*}$ & 0.436 \\
\hline & Male & $41.70^{*}$ & $30.80^{*}$ & 0.990 \\
\hline Age (min; max) & & $77.68^{\dagger}(65 ; 94)$ & $79.1^{\dagger}(65 ; 101)$ & 0.940 \\
\hline Years since diagnosis (min; $\max$ ) & & NA & $5.29^{\dagger}(1 ; 12)$ & - \\
\hline \multirow[t]{3}{*}{ Severity of $A D$} & Mild & NA & $20^{*}$ & - \\
\hline & Moderate & NA & $28.30^{*}$ & - \\
\hline & Severe & NA & $51.70^{*}$ & - \\
\hline \multirow[t]{2}{*}{ Systemic pathologies } & Yes & $58.30^{*}$ & $59.20^{*}$ & 0.860 \\
\hline & No & $41.70^{*}$ & $40.80^{*}$ & 0.841 \\
\hline \multirow[t]{2}{*}{ Pharmacologic therapy } & Yes & $59.20^{*}$ & $91.70^{*}$ & $<0.001$ \\
\hline & No & $40.80^{*}$ & $8.30^{*}$ & $<0.001$ \\
\hline \multirow[t]{3}{*}{ ASA classification } & ASA 1 & $56.30^{*}$ & $0^{*}$ & $<0.001$ \\
\hline & ASA 2 & $38.80^{*}$ & $79.20^{*}$ & $<0.001$ \\
\hline & ASA 3 & $4.90^{*}$ & $20.80^{*}$ & $<0.001$ \\
\hline Edentulism & & $31.6^{*}$ & $39.16^{*}$ & 0.102 \\
\hline
\end{tabular}

${ }^{*}$ Data are in percentage; ${ }^{\dagger}$ Data are median in years 
Table 2: Epidemiological indices in the control and AD groups

\begin{tabular}{llll}
\hline $\begin{array}{l}\text { Epidemiological } \\
\text { indices }\end{array}$ & $\begin{array}{l}\text { Control }(n=71 \\
\text { of 103) }\end{array}$ & $\begin{array}{l}A D(n=73 \\
\text { of 120) }\end{array}$ & Significance \\
\hline Decayed teeth (D) & $1.17 \pm 0.93^{*}$ & $1.53 \pm 0.73^{*}$ & 0.005 \\
Filled teeth (F) & $1.66 \pm 0.49^{*}$ & $0.64 \pm 0.32^{*}$ & $<0.001$ \\
$\begin{array}{l}\text { Remaining natural } \\
\text { teeth (T) }\end{array}$ & $12.41 \pm 5.21^{*}$ & $9.15 \pm 4.82^{*}$ & $<0.001$ \\
DF/T & & & \\
$\mathrm{CPI}^{\dagger}$ & $0.80 \pm 0.70^{*}$ & $0.20 \pm 0.15^{*}$ & $<0.001$ \\
$\mathrm{GI}$ & $1.83 \pm 0.06^{*}$ & $2.15 \pm 0.74^{*}$ & $<0.001$ \\
\hline
\end{tabular}

*Data are mean \pm standard deviation; ${ }^{\dagger}$ Decayed and filled teeth/ remaining natural teeth ratio

detected between control and AD groups: most variables presented statistically higher values in the study group, in particular D teeth, CPI, and GI ( $\mathrm{p}=0.005, \mathrm{p}<0.001$, and $\mathrm{p}<0.001$ respectively). The mean number of $\mathrm{F}$ teeth and remaining $\mathrm{T}$ teeth and the $\mathrm{DF} / \mathrm{T}$ ratio were significantly lower in $\mathrm{AD}$ patients compared with the control group $(p<0.001)$. Mean values and standard deviations of the indices used in the study are displayed in Table 2.

Table 3 shows a description of AD subgroups (institutionalized and noninstitutionalized) and a t-test is used for comparison. Each subgroup is composed of 60 subjects. Institutionalized patients' median age was 80.34 years (min: 65, max: 101) and was not significantly different than age in noninstitutionalized patients $(p=0.745)$. This group comprised 47 females (78.33\%) and 13 males (21.67\%). The noninstitutionalized subgroup's median age was 77.93 years (min: 66, max: 92) and included 37 females $(61.66 \%)$ and 23 males $(38.34 \%)$. Gender was equally distributed between the two subgroups ( $\mathrm{p}=$ $0.725, p=0.973$ ). The average since the diagnosis of AD in institutionalized patients was 5.93 years (min: 2, max: 12), which is not significantly different from the noninstitutionalized subgroup $(p=0.659)$. The severity of $\mathrm{AD}$ was mild in $1.7 \%$ of the institutionalized subgroup and statistically lower than the percentage (38.40\%) of noninstitutionalized patients with a mild AD diagnosis $(\mathrm{p}<0.001)$; a statistically greater number of moderate $\mathrm{AD}$ patients was present in the institutionalized subgroup $(38.4 \%, \mathrm{p}<0.001)$. Similarly, the percentage of severe cases of AD was significantly higher in the institutionalized subgroup $(76.67 \%, \mathrm{p}<0.001)$. The institutionalized subgroup was significantly more affected by additional systemic pathologies $(\mathrm{p}<0.001)$ and all the subjects were under pharmacological therapy $(100 \%)$. However, the percentage of noninstitutionalized patients assuming pharmacologic therapy was not significantly different $(85 \%, \mathrm{p}=$ $0.277)$. Ninety percent of noninstitutionalized AD patients were classified as ASA 2, which is significantly higher than ASA 2 in the institutionalized subgroup $(\mathrm{p}<0.001)$. A significantly higher percentage of institutionalized AD patients were classified as ASA $3(35 \%, \mathrm{p}<0.001)$. The percentage of edentulous patients is significantly higher $(55 \%, \mathrm{p}<0.001)$ in the institutionalized subgroup.

Epidemiological indices for both the institutionalized and noninstitutionalized subgroups are shown in Table 4. The mean number of $D$ teeth was not significantly different between the two subgroups $(p=0.145)$. The average number of $\mathrm{F}$ teeth is greater in noninstitutionalized $\mathrm{AD}$ patients $(\mathrm{p}<0.001)$. The average number of remaining $\mathrm{T}$ teeth is greater in noninstitutionalized patients; however, it is not statistically significant ( $p=0.077$ ). On the contrary, the mean DF/T ratio, the CPI, and the GI were significantly higher in the institutionalized AD subgroup ( $\mathrm{p}<0.001, \mathrm{p}=0.024$, and $\mathrm{p}<0.001$ respectively).

Table 5 shows the Spearman's correlation coefficient (r) between oral health status of $\mathrm{AD}$ patients and $\mathrm{AD}$

Table 3: Characteristics of AD subgroups

\begin{tabular}{|c|c|c|c|c|}
\hline & & \multicolumn{2}{|c|}{$A D(n=120)$} & \multirow[b]{2}{*}{ Significance } \\
\hline & & Institutionalized $(n=60)$ & Noninstitutionalized $(n=60)$ & \\
\hline \multirow[t]{2}{*}{ Gender } & Female & $78.33^{*}$ & $61.66^{*}$ & 0.725 \\
\hline & Male & $21.67^{*}$ & $38.34^{*}$ & 0.973 \\
\hline Age (min; max) & & $80.34^{\dagger}(65 ; 101)$ & $77.90^{\dagger}(66 ; 92)$ & 0.745 \\
\hline Years from diagnosis (min; max) & & $5.93^{\dagger}(2 ; 12)$ & $4.65^{\dagger}(1 ; 10)$ & 0.659 \\
\hline \multirow[t]{3}{*}{ Severity of $A D$} & Mild & $1.70^{*}$ & $38.40^{*}$ & $<0.001$ \\
\hline & Moderate & $18.30^{*}$ & $38.40^{*}$ & $<0.001$ \\
\hline & Severe & $80.00^{*}$ & $23.20^{*}$ & $<0.001$ \\
\hline \multirow[t]{2}{*}{ Systemic pathologies } & Yes & $76.67^{*}$ & $56.67^{*}$ & $<0.001$ \\
\hline & No & $23.34^{*}$ & $43.33^{*}$ & $<0.001$ \\
\hline \multirow[t]{2}{*}{ Pharmacologic therapy } & Yes & $100^{*}$ & $85.00^{*}$ & 0.277 \\
\hline & No & $0^{*}$ & $15.00^{*}$ & 0.310 \\
\hline \multirow[t]{3}{*}{ ASA classification } & ASA 1 & $0^{*}$ & $0^{*}$ & 0.409 \\
\hline & ASA 2 & $65.00^{*}$ & $90.00^{*}$ & $<0.001$ \\
\hline & ASA 3 & $35.00^{*}$ & $10.00^{*}$ & $<0.001$ \\
\hline Edentulism & & $55^{\star}$ & $23.33^{*}$ & $<0.001$ \\
\hline
\end{tabular}

${ }^{*}$ Data are percentage; ${ }^{\dagger}$ Data are median in years 
Oral Health Status in Alzheimer's Disease Patients

Table 4: Epidemiological indices in the $A D$ subgroups

\begin{tabular}{lllr}
\hline Epidemiological indices & $\begin{array}{l}\text { Institutionalized } A D \\
(n=27 \text { of } 60)\end{array}$ & $\begin{array}{l}\text { Noninstitutionalized } \\
A D(n=46 \text { of } 60)\end{array}$ & Significance \\
\hline Decayed teeth $(\mathrm{D})$ & $1.61 \pm 0.51^{*}$ & $1.45 \pm 0.46^{*}$ & 0.145 \\
Filled teeth $(\mathrm{F})$ & $0.46 \pm 0.15^{*}$ & $0.88 \pm 0.51^{*}$ & $<0.001$ \\
Remaining natural teeth (T) & $8.98 \pm 5.58^{*}$ & $12.41 \pm 5.59^{*}$ & 0.077 \\
DF/T & $0.30 \pm 0.15^{\star}$ & $0.16 \pm 0.08^{*}$ & $<0.001$ \\
CPI & $2.33 \pm 0.91^{*}$ & $1.97 \pm 0.82^{*}$ & 0.024 \\
GI & $2.70 \pm 0.66^{*}$ & $2.23 \pm 0.70^{*}$ & $<0.001$ \\
\hline
\end{tabular}

*Data are mean \pm standard deviation; ${ }^{\dagger}$ Decayed and filled teeth/remaining natural teeth ratio

Table 5: Correlation between oral health status and AD severity

\begin{tabular}{llllllll}
\hline $\begin{array}{l}\text { Correlation coefficient }(r) \\
\text { (two-tailed) } n=120\end{array}$ & $A D$ severity & $D$ & $F$ & $T$ & $D F / T$ & $C P I$ & $G I$ \\
\hline AD severity & 1.000 & -0.121 & $-0.350^{*}$ & $-0.372^{*}$ & $-0.247^{*}$ & $-0.270^{*}$ & $-0.192^{*}$ \\
D & -0.121 & 1.000 & $0.447^{*}$ & $0.536^{*}$ & $0.497^{*}$ & $0.541^{*}$ & $0.520^{*}$ \\
F & $-0.350^{*}$ & $0.447^{*}$ & 1.000 & $0.576^{*}$ & $0.228^{*}$ & $0.336^{*}$ & $0.245^{*}$ \\
T & $-0.372^{*}$ & $0.536^{*}$ & $0.576^{*}$ & 1.000 & $0.340^{*}$ & $0.679^{*}$ & $0.633^{*}$ \\
DF/T & $-0.247^{*}$ & $0.497^{*}$ & $0.228^{*}$ & $0.340^{*}$ & 1.000 & $0.370^{*}$ & $0.400^{*}$ \\
CPI & $-0.270^{*}$ & $0.541^{*}$ & $0.336^{*}$ & $0.679^{*}$ & $0.370^{*}$ & 1.000 & $0.886^{*}$ \\
GI & $-0.192^{*}$ & $0.520^{*}$ & $0.245^{*}$ & $0.633^{*}$ & $0.400^{*}$ & $0.886^{*}$ & 1.000 \\
\hline
\end{tabular}

*Significant at a $p$-value $\leq 0.05$

severity. The results show a significant, however weak, negative correlation of the different indices used in the study and the severity of AD. The values of $\mathrm{r}$ ranged from -0.121 to -0.372 .

\section{DISCUSSION}

The results of this study revealed that AD patients present a higher prevalence of oral pathology compared with healthy subjects. Our data are in line with the results of other groups of AD patients coming from different geographical areas. The indices used in this study (DMFT, CPI, GI) indicated significantly high values of caries and periodontal disease, comparable with those observed in epidemiological surveys on populations suffering from senile dementia. ${ }^{15,17,18}$ The high prevalence of carious lesions in this particular population is linked to several factors: drug-induced xerostomia, patients' inability in performing proper oral hygiene, and dependence on caregivers. $^{14}$

A high prevalence of tooth loss, related to the severity of the pathology, was a major finding in our study. This could be explained by the high values of periodontal indices (CPI and GI) and prevalence of carious lesions affecting AD patients, in agreement with the findings from multiple studies on oral health of patients with AD. ${ }^{15,17,18}$

The obtained results indicate how the indices of caries and periodontal disease are related to the degree of severity of AD. Probably, the severity of the disease is positively correlated with poor oral health conditions and this could change the oral health status. These findings were not reported in a study conducted on 50 patients with AD that reports the absence of statistically significant relationships between AD severity and oral health status. ${ }^{19}$ These findings could be related to a small sample group, a lack of distinction between the severity of the disease, and poorer oral health conditions of the patients.

The data suggest that patients in institutions are more prone to tooth decay but are less frequently subjected to dental conservative therapy compared with those of noninstitutionalized (i.e., aging at home). The average number of remaining teeth is significantly lower in the institutionalized group when compared with noninstitutionalized AD patients and the percentage of totally edentulous patients is doubled in the institutionalized group. These findings reflect the higher severity of $\mathrm{AD}$ in the institutionalized group that is likely responsible for a worsening of the oral health status and a tendency toward tooth extractions in this compromised population, which usually presents challenges during dental chair side management.

The preservation of a functional stomatognathic system is an important goal in the management of patients in general, and AD patients in particular, to maintain functions, such as chewing, speech, and esthetics in order to improve the quality of life of patients. A multidisciplinary team and multiple figures are usually necessary for implementation of specific preventive and treatment strategies in AD patients: the family of the patient and/ or caregivers, in addition to the treating physicians and the dentist. As the severity of the disease increases, AD patients become more compromised both medically and dentally; this is worsened by cognitive impairments and 
lack of cooperation. From a dental standpoint, the oral hygiene maneuvers become difficult to be performed by the patients alone and usually they become dependent on a family member and/or caregiver.

Alzheimer's disease patients require specific management due to their poor cooperation. Oral hygiene maneuvers performed by nurses and caregivers may be perceived as a threat by patients with dementia. ${ }^{20}$ The frequency of episodes of noncooperation behavior during dental treatment in AD patients is directly related to the severity of dementia and to a higher rates of CPI and GI. ${ }^{21}$ The decline of cognitive function in patients with AD was associated with a marked decrease of personal and oral health care and an increase of denture stomatitis. ${ }^{22}$ Underdiagnosis and underestimation of pain, although equally perceived, is a common finding in dementia patients. ${ }^{23,24}$

The noneffective management of oral health care from the staff and caregivers may be attributable to high workloads, "difficult" patients, and lack of information and education on oral hygiene techniques. Appropriate educational programs and customized guidelines for family members and/or caregivers of $\mathrm{AD}$ patients are strongly encouraged. Detailed questionnaires regarding the basic oral health information may be administered to family members and/or caregivers in order to plan tailored educational programs. The British Society of Gerodontology presented guidelines that aimed at guiding health professionals, caregivers, and family members of patients with dementia. However, there are currently no specific guidelines for patients with AD. ${ }^{25}$ Moreover, professional oral health controls and hygiene protocols could be programmed, excluding intervention in general anesthesia and sedation due to their neural toxicity. $^{26,27}$

Investigation of the subjective oral health care needs in the elderly population may be implemented to personalize professional efforts for the needs of the patients. Similarly to what we implemented in our study, oral health status was historically assessed by epidemiological surveys using clinical indicators. Specific measures of oral health-related quality of life are also widely used. ${ }^{28}$ A useful tool to predict the need for oral examination at an individual level in elderly individuals is the Geriatric Oral Health Assessment Index. ${ }^{29}$ Further studies that assess the subjective and objective quality of life of AD patients are strongly encouraged.

\section{CONCLUSION}

- All indicators of oral pathology evaluated were significantly higher in the group of patients with AD compared with the control group.
- Institutionalized AD subjects presented an inferior number of natural teeth compared with the noninstitutionalized subgroup.

- The severity of AD has a negative effect on the oral health status, and the type of institutionalization further exacerbates it.

- Further research is needed to investigate oral health care interventions needed in patients with AD.

- It would be beneficial to introduce trained professional figures in specialized elderly institutions (e.g., dentist and dental hygienist) for regular follow-up visits and periodic professional oral hygiene procedures in addition to oral diagnosis and treatment. This task has to be in coordination with the treating physician, family members, and/or caregivers.

\section{CLINICAL SIGNIFICANCE}

Once the diagnosis of AD or dementia is set, the patient's oral health status must be evaluated. In fact, it must be considered that it will worsen due to their cognitive and manual ability reduction. For this reason, oral hygiene protocol should be started, in order to preserve the functional ability of the patients. In particular, this is mandatory for institutionalized patients who present a higher severity of the disease. Their caregivers should be trained on oral health care or an oral hygienist should assist them in order to prevent a rapid worsening of the patient's oral functions until the end.

\section{REFERENCES}

1. Sosa-Ortiz AL, Acosta-Castillo I, Prince MJ. Epidemiology of dementias and Alzheimer's disease. Arch Med Res 2012 Nov;43(8):600-608.

2. Prince M, Bryce R, Albanese E, Wimo A, Ribeiro W, Ferri CP. The global prevalence of dementia: a systematic review and meta-analysis. Alzheimers Dement 2013 Jan;9(1):63-75.

3. Mayeux R, Stern Y. Epidemiology of Alzheimer disease. Cold Spring Harbor Perspect Med 2012 Aug;2(8):pii:a006239.

4. Nowrangi MA, Rao V, Lyketsos CG. Epidemiology, assessment, and treatment of dementia. Psychiatr Clin North Am 2011 Jun;34(2):275-294.

5. Reitz C, Brayne C, Mayeux R. Epidemiology of Alzheimer disease. Nat Rev Neurol 2011 Mar;7(3):137-152.

6. Matthews FE, Arthur A, Barnes LE, Bond J, Jagger C, Robinson L, Brayne C, Medical Research Council Cognitive Function and Ageing Collaboration. A two-decade comparison of prevalence of dementia in individuals aged 65 years and older from three geographical areas of England: results of the Cognitive Function and Ageing Study I and II. Lancet 2013 Oct;382(9902):1405-1412.

7. Christensen $\mathrm{K}$, Thinggaard M, Oksuzyan A, Steenstrup T, Andersen-Ranberg K, Jeune B, McGue M, Vaupel JW. Physical and cognitive functioning of people older than 90 years: a comparison of two Danish cohorts born 10 years apart. Lancet 2013 Nov;382(9903):1507-1513. 
8. Schrijvers EM, Verhaaren BF, Koudstaal PJ, Hofman A, Ikram MA, Breteler MM. Is dementia incidence declining? Trends in dementia incidence since 1990 in the Rotterdam Study. Neurology 2012 May;78(19):1456-1463.

9. Rocca WA, Petersen RC, Knopman DS, Hebert LE, Evans DA, Hall KS, Gao S, Unverzagt FW, Langa KM, Larson EB, et al. Trends in the incidence and prevalence of Alzheimer's disease, dementia, and cognitive impairment in the United States. Alzheimers Dement 2011 Jan;7(1):80-93.

10. Reitz C, Mayeux R. Alzheimer disease: epidemiology, diagnostic criteria, risk factors and biomarkers. Biochem Pharmacol 2014 Apr;88(4):640-651.

11. Sparks Stein P,Steffenc MJ,Smith C, Jicha G, EbersoleJL,AbnerE, Dawson III D. Serum antibodies to periodontal pathogens are a risk factor for Alzheimer's disease. Alzheimers Dement 2012 May;8(3):196-203.

12. Pazos P, Leira Y, Domínguez C, Pías-Peleteiro JM, Blancoa J, Aldrey JM. Asociación entre enfermedad periodontal y demencia. Revisión de la bibliografía. Neurología 2016: Article in press.

13. Miklossy J. Emerging roles of pathogens in Alzheimer disease. Exp Rev Mol Med 2011 Sep;20:13:e30.

14. Shetty SR, Bhowmick S, Castelino R, Babu S. Drug induced xerostomia in elderly individuals: an institutional study. Contemp Clin Dent 2012 Apr-Jun;3(2):173-175.

15. Cicciù $M$, Matacena $G$, Signorino $F$, Brugaletta $A$, Cicciù $A$, Bramanti E. Relationship between oral health and its impact on the quality life of Alzheimer's disease patients: a supportive care trial. Int J Clin Exp Med 2013;6(9):766-772.

16. World Health Organization. Oral health surveys: basic methods. 4th ed. Geneva: ORH/EPID; 1997.

17. Stewart R, Weyant RJ, Garcia ME, Harris T, Launer LJ, Satterfield S, Simonsick EM, Yaffe K, Newman AB. Adverse oral health and cognitive decline: the health, aging and body composition study. J Am Geriatr Soc 2013 Feb;61(2):177-184.

18. Kaye EK, Valencia A, Baba N, Spiro A, Dietrich T, Garcia RI. Tooth loss and periodontal disease predict poor cognitive function in older men. J Am Geriatr Soc 2010 Apr;58(4):713-718.
19. Machado MC. Oral health of Alzheimer's patients in Sao Josè dos Campos, Brazil. Geriatr Gerontol Int 2012 Apr;12(2): 2265-2270.

20. Jablonski RA,KolanowskiA, Therrien B,Mahoney EK, KassabC, Leslie DL. Reducing care-resistant behaviors during oral hygiene in persons with dementia. BMC Oral Health 2011 Nov;11:30.

21. Philip P, Rogers C, Kruger E, Tennant M. Oral hygiene care status of elderly with dementia and in residential aged care facilities. Gerodontology 2012 Jun;29(2):e306-e311.

22. Ribeiro GR, Riani Costa JL, Bovi Ambrosano GM, Rodrigues Garcia RCM. Oral health of the elderly with Alzheimer's disease. Oral Surg Oral Med Oral Pathol Oral Radiol 2012 Sep;114(3):338-343.

23. Achterberg WP, Pieper MJ, van Dalen-Kok AH, de Waal MW, Husebo BS, Lautenbacher S, Kunz M, Scherder EJ, Corbett A. Pain management in patients with dementia. Clin Interv Aging 2013;8:1471-1482.

24. Herr K. Pain assessment strategies in older patients. J Pain 2011 Mar;12(3 Suppl 1):S3-S13.

25. Sonde L, Emami A, Kiljunen H. Care providers' perceptions of the importance of oral care and its performance within everyday caregiving for nursing home residents with dementia. Scan J Caring Sci 2011 Mar;25(1):92-99.

26. Oomens MA, Booij LH, Baart JA. The risk of general anesthesia and sedation in older people. Ned Tijdschr Tandheelkd 2015 Dec;122(12):674-679.

27. Campos CH, Ribeiro GR, Rodrigues Garcia RC. Oral healthrelated quality of life in mild Alzheimer: patient versus caregiver perception. Spec Care Dentist 2016 Sep;36(5):271-276.

28. Zuluaga DJ, Montoya JA, Contreras CI, Herrera RR. Association between oral health, cognitive impairment and oral health-related quality of life. Gerodontology 2012 Jun;29(2):667-673.

29. ElOsta N, Tubert-JeanninS, Hennequin M,Abboud Naaman NB, El Osta L, Geahchan N. Comparison of the OHIP-14 and GOHAI as measures of oral health among elderly in Lebanon. Health Qual Life Outcomes 2012 Oct;10:131. 